\title{
POTENCIAL DE REDUÇÃO DO CONSUMO DE ÁGUA PARA DIFERENTES TIPOLOGIAS ARQUITETÔNICAS NA BACIA DO RIO TRAPICHEIRO, RJ
}

\section{Potential water consumption decrease on different architectural typologies in the Trapicheiro Watershed, RJ}

\author{
OKUMURA, Celí Kiyomi ${ }^{1}$; CARVALHO, Fabiana Ferreira ${ }^{2}$; RODRIGUES, Silvana Barbeitas \\ Lourenço $^{3}$; GUIMARÃES, Luciana Fernandes ${ }^{4}$; VERÓL, Aline Pires ${ }^{5}$
}

Recebido em 15 de jullho de 2021, aprovado em 06 de setembro de 2021, publicado em 18 de novembro de 2021

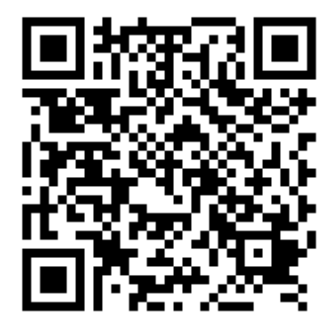

Palavras-chave:

Sistemas prediais,

Conservação da água,

Tipologias

arquitetônicas.

Keywords:

Building systems,

Water conservation,

Architectural typologies.
RESUMO: A concepção dos sistemas prediais hidrossanitários assume um papel relevante na sustentabilidade ambiental edilícia. No tocante à conservação de água nas edificações, diferentes ações podem otimizar a operação desses sistemas, como a adoção de dispositivos economizadores e o uso de fontes hídricas não convencionais para fins não potáveis. Tais medidas reduzem o consumo de água potável, beneficiando economicamente $o$ usuário e diminuindo a pressão sobre a disponibilidade hídrica na escala urbana. Neste contexto, este trabalho tem por objetivo avaliar e comparar o potencial de redução do consumo de água em edificações, considerando três tipologias arquitetônicas de uso residencial na Bacia do Rio Trapicheiro, município do Rio de Janeiro. A partir do estudo, verificou-se que o uso de dispositivos economizadores pode ser uma solução sustentável generalizada na construção civil, independente da tipologia arquitetônica. Por outro lado, o aproveitamento de água de chuva depende da área de captação. Além disso, observou-se, também, que o potencial de economia gerado por unidade domiciliar decresce com a verticalização da edificação. Por fim, observa-se que o tempo de retorno obtido para a substituição dos aparelhos tradicionais é aceitável para classe média brasileira, sendo necessário avaliar mecanismos de incentivo para classes menos favorecidas.

ABSTRACT: The plumbing systems design plays an important role in building environmental sustainability. Regarding water conservation in buildings, distinct initiatives can optimize the operation of these systems, such as the adoption of lowflow fittings and the use of non-conventional water sources for non-potable purposes. These measures reduce drinking water consumption, economically benefiting the consumer and reducing the pressure on water availability at the urban scale. In this context, this work aims to evaluate and compare the potential for reducing water consumption in buildings, considering three architectural typologies of residential use in the Trapicheiros Watershed, in the City of Rio de Janeiro. The study indicates that the use of low-flow fittings can be a widespread sustainable solution in civil construction, regardless of the architectural typology. On the other hand, the use of rainwater depends on the catchment area. In addition, it was also observed that the potential for savings generated by households decreases with the building verticalization. Finally, it is observed that the payback obtained for the replacement of traditional sanitary fittings is acceptable for the Brazilian middle class, and it is necessary to evaluate incentive mechanisms for those from lower-income classes.

\section{CONTATO DOS AUTORES:}

${ }^{1}$ OKUMURA, Celí Kiyomi: Programa de Pós-Graduação em Arquitetura, Faculdade de Arquitetura de Urbanismo, Universidade Federal do Rio de Janeiro, celi.okumura@fau.ufrj.br

${ }^{2}$ CARVALHO, Fabiana Ferreira: Programa de Pós-Graduação em Arquitetura, Faculdade de Arquitetura de Urbanismo, Universidade Federal do Rio de Janeiro, fabiana.carvalho@fau.ufrj.br 


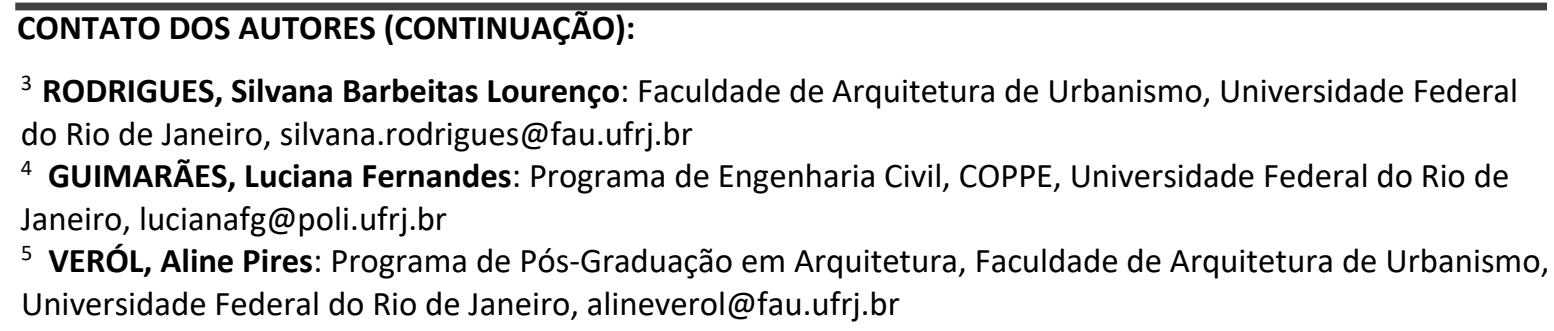

XIV SIMPÓSIO NACIONAL DE SISTEMAS PREDIAIS (SISPRED 2021)

\section{INTRODUÇÃO}

A concepção dos sistemas prediais hidrossanitários assume um papel relevante na sustentabilidade ambiental edilícia. No tocante à conservação de água nas edificações, existem diferentes ações que otimizam a operação desses sistemas, dentre os quais pode-se destacar a medição individualizada (SALGADO, 2008), que auxilia na conscientização do usuário sobre o real consumo da edificação, a substituição de aparelhos tradicionais por dispositivos economizadores (DE), reduzindo a demanda da edificação, e o uso de fontes hídricas não convencionais para fins não potáveis, usualmente denominadas alternativas de gestão da oferta, como o aproveitamento de água de chuva de coberturas (ACC). Tais medidas reduzem o consumo de água potável, beneficiando economicamente o usuário e, na escala urbana, diminuindo a pressão sobre a disponibilidade hídrica. Além disso, promovem menor consumo de energia, reduzindo custos de tratamento e distribuição de água potável, bem como, contribuem para a preservação de recursos naturais (DA SILVA et al., 2020).

Portanto, pode-se dizer que os cuidados com os sistemas prediais hidrossanitários de uma edificação revertem para o bom funcionamento da cidade, apontando para um caminho de desenvolvimento, pois é parte da indústria da construção que desempenha papel fundamental no crescimento das cidades (BALOI, 2003). Nesse contexto, em apoio ao conceito de desenvolvimento sustentável, publicou-se a Agenda 21 para construção sustentável em 1999 e, mais tarde, a Nova Agenda Urbana (2016), com o intuito de promover a adoção de processos de construção sustentável que envolvam novos procedimentos de projeto e de materiais, operação amigável e manutenção orientada ambientalmente, para ajudar a mitigar as perdas de recursos naturais e os desafios das mudanças climáticas (CIB; UNEP-IETC, 2002).

No Brasil, a publicação das normas NBR 16.782 (ABNT, 2019b), que trata da conservação de água em edifícios novos e existente e NBR 16.783 (ABNT, 2019c), que aborda o uso de fontes alternativas não potáveis em edificações, reforçam a preocupação no âmbito da construção civil nacional.

Nesse sentido, este trabalho tem por objetivo avaliar e comparar o potencial de redução do consumo de água em edificações considerando a adoção de DE ou do aproveitamento de ACC. Para esta análise, foram selecionadas três tipologias arquitetônicas de uso residencial (TAUR) em um ambiente urbano consolidado de forma a verificar se há ou não influência da tipologia no potencial de economia de água potável considerando as alternativas propostas. Como limitação, o presente estudo avalia a utilização de uma única ação sustentável, ou seja, na alternativa com o aproveitamento de ACC não é considerada a substituição dos aparelhos tradicionais. Além disso, na comparação inicial é considerado o potencial de aproveitamento 
de ACC (disponibilidade teórica de água de chuva) sem a especificação dos pontos de consumo de água não potável na edificação.

\section{MÉTODO}

Este trabalho foi desenvolvido tendo como caso de estudo a Bacia do Rio Trapicheiro, localizada na Zona Norte do município do Rio de Janeiro. Nesse contexto, para avaliar o potencial de redução do consumo de água na bacia, foram adotadas as seguintes etapas:

a) Seleção de três TAUR;

b) Análise do consumo hídrico mensal de cada tipologia:

b.1) Consumo total (VT), considerando-se o consumo médio de água de 2015 a 2019 no MRJ (SNIS, $[s . d]$.$) , a quantidade de economias e a média de pessoas por domicílio$ (IBGE, [s. d.]);

b.2) Distribuição do consumo por pontos de utilização, baseando-se nos percentuais descritos no PNCDA (1998, p. 32);

c) Estimativa do potencial de redução do consumo de água potável propondo duas alternativas:

c.1) Substituição de aparelhos tradicionais (bacias sanitárias, chuveiros, torneiras do lavatório, da cozinha e da área de serviço) por DE. A economia de água foi estimada a partir da média aritmética do percentual de redução de consumo informado por quatro fabricantes para aparelhos semelhantes. Os demais aparelhos foram desconsiderados, adotando-se volumes fixos pertinentes às atividades;

c.2) Aproveitamento de ACC, baseando-se na área do telhado das tipologias e nos dados pluviométricos do Posto da Tijuca do período de 1997 a 2018 (ALERTA RIO, [s. d.]). Para essa alternativa, foi considerada exclusivamente a disponibilidade teórica de ACC para cada tipologia - NBR 15.527 (ABNT, 2019a), sem a especificação das demandas por água não potável, de modo a permitir a comparação com a economia gerada por DE.

d) Fatura mensal da alternativa que apresentou maior potencial de redução do consumo por TAUR, considerando-se 30 dias, as faixas de cobrança praticada pela CEDAE ([s.d.]);

e) Análise comparativa dos resultados de redução tarifária e de consumo.

\section{RESULTADOS E DISCUSSÃO}

\subsection{Caracterização do caso de estudo}

O bairro Tijuca, localizado na Zona Norte do Município do Rio de Janeiro (MRJ), foi selecionado por sua relevância histórica na expansão urbana local, cujo início ocorreu no final do século XIX, consolidando-se ao longo do século XX (QUEIROZ et al., 2008). Portanto, nesse ambiente urbano foram selecionadas as três tipologias arquitetônicas mais recorrentes, apresentadas na Figura 1, para avaliar o desempenho da aplicação das duas alternativas propostas para reduzir o consumo de água.

Como premissa para seleção das tipologias, optou-se por avaliar edifícios de uso residencial, sendo um unifamiliar e dois multifamiliares com gabaritos de quatro e onze pavimentos que 
apresentam a mesma composição de áreas molhadas: dois banheiros, uma cozinha, uma área de serviço e um banheiro de serviço. Outras características das TAUR são apresentadas na Tabela 1.

Figura 1 - Localização das TAUR na Bacia do Rio Trapicheiro - RJ
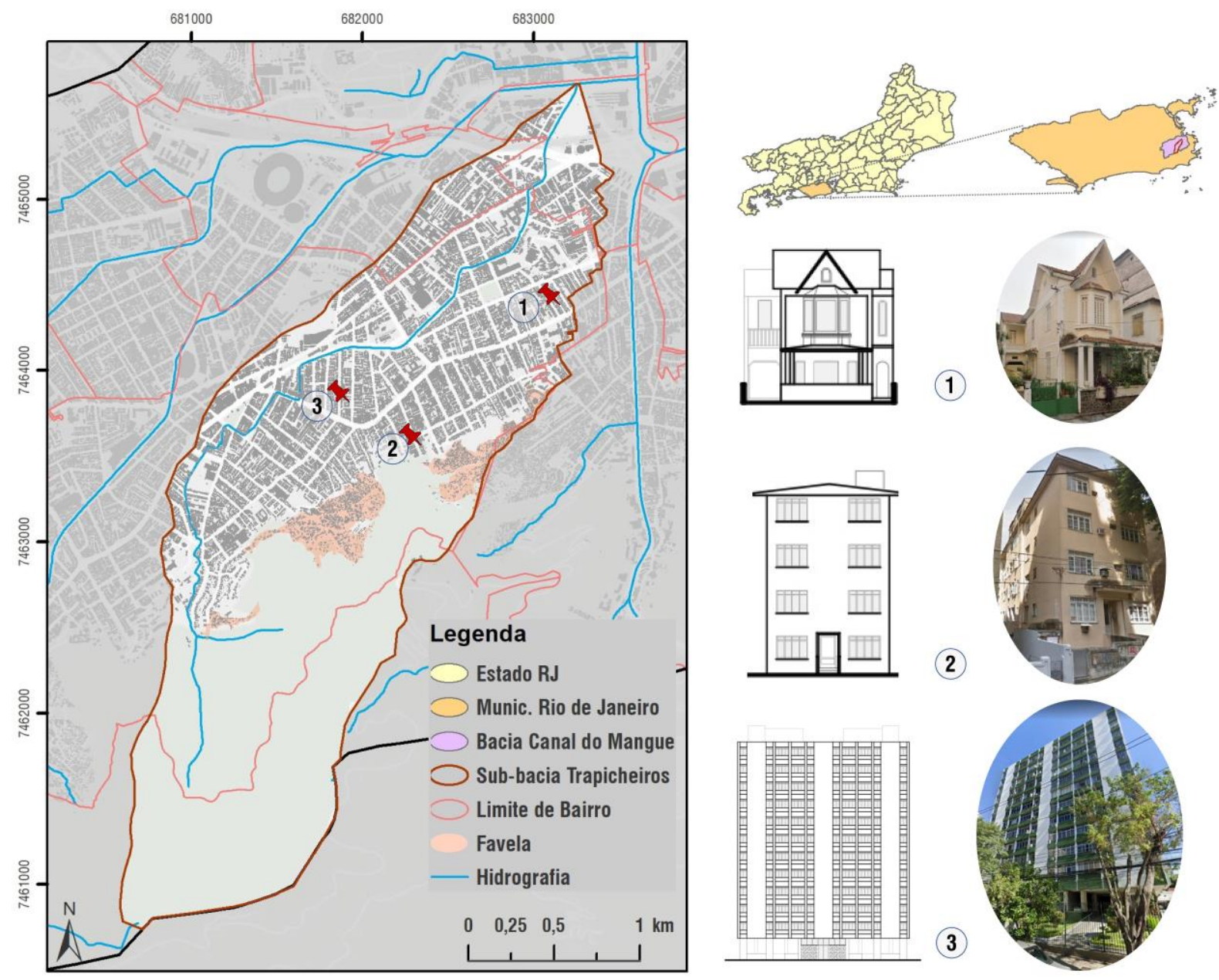

(1)
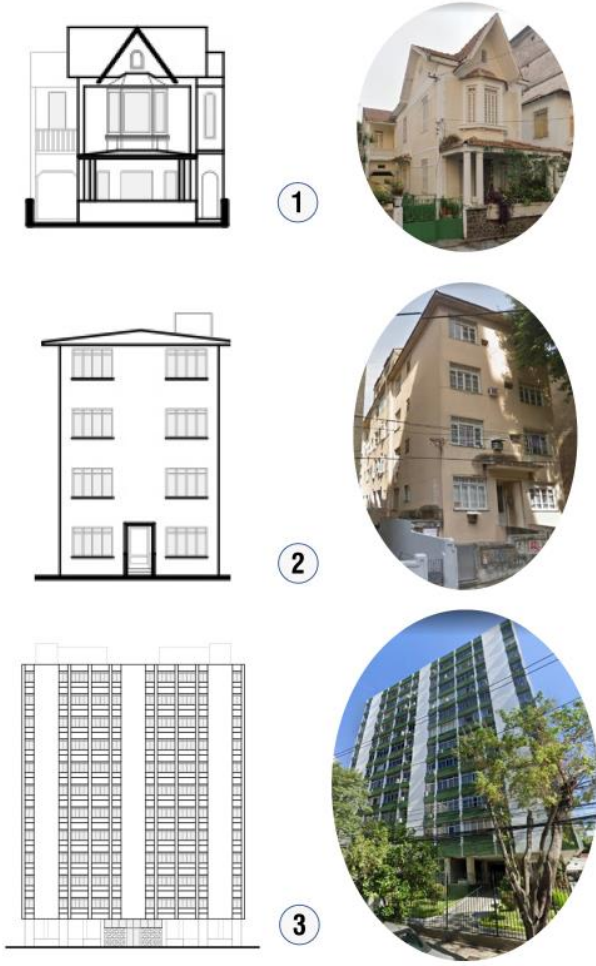

Fonte: Autoria própria (2021).

Tabela 1 - Características das TAUR

\begin{tabular}{|c|c|c|c|c|c|c|c|}
\hline \multirow[b]{2}{*}{ TAUR } & \multirow[b]{2}{*}{ Economias } & \multicolumn{4}{|c|}{ Área $\left(m^{2}\right)$} & \multirow{2}{*}{$\begin{array}{l}\text { Consumo } \\
\left(\mathrm{m}^{3} / \mathrm{mês}\right)\end{array}$} & \multirow{2}{*}{$\begin{array}{c}\text { Tarifa Mensa } \\
\text { (R\$) }\end{array}$} \\
\hline & & Terreno & Telhado & Jardim & $\begin{array}{c}\text { Piso } \\
\text { externo }\end{array}$ & & \\
\hline Sobrado & 1 & 171 & 115 & 2 & 53 & 27,45 & 392,94 \\
\hline Edifício 4 pav. & 8 & 360 & 240 & 6 & 114 & 219,60 & $3.143,50$ \\
\hline Edifício 11 pav. & 110 & 2140 & 1000 & 100 & 940 & $3.019,50$ & $43.223,16$ \\
\hline
\end{tabular}

Fonte: Autoria própria (2021). 


\subsection{Resultados}

Com a substituição dos aparelhos tradicionais por DE observa-se um potencial de redução do consumo de cerca de $50 \%$, independentemente da TAUR, conforme apresentado na Figura 2. Por outro lado, o aproveitamento da ACC está limitado à precipitação média e à área de captação no telhado, resultando em potenciais de redução no consumo de água potável variáveis em função da TAUR. Apesar de ocorrer um incremento na área do telhado nas tipologias multifamiliares, em especial no edifício de 11 pavimentos, esse aumento não é proporcional à quantidade de economias. Com isso, o potencial de redução do consumo por unidade decresce com a verticalização da edificação. As Figuras 2 e 3 apresentam os resultados obtidos em termos de redução do consumo e água potável na edificação e por unidade residencial, respectivamente. Além disso, o potencial de aproveitamento de ACC no mês de menor índice pluviométrico (agosto) também é apresentado considerando que o uso do potencial médio demanda reservatórios de maior capacidade para regularizar a vazão.

Figura 2 - Potencial de redução do consumo de água potável da edificação.

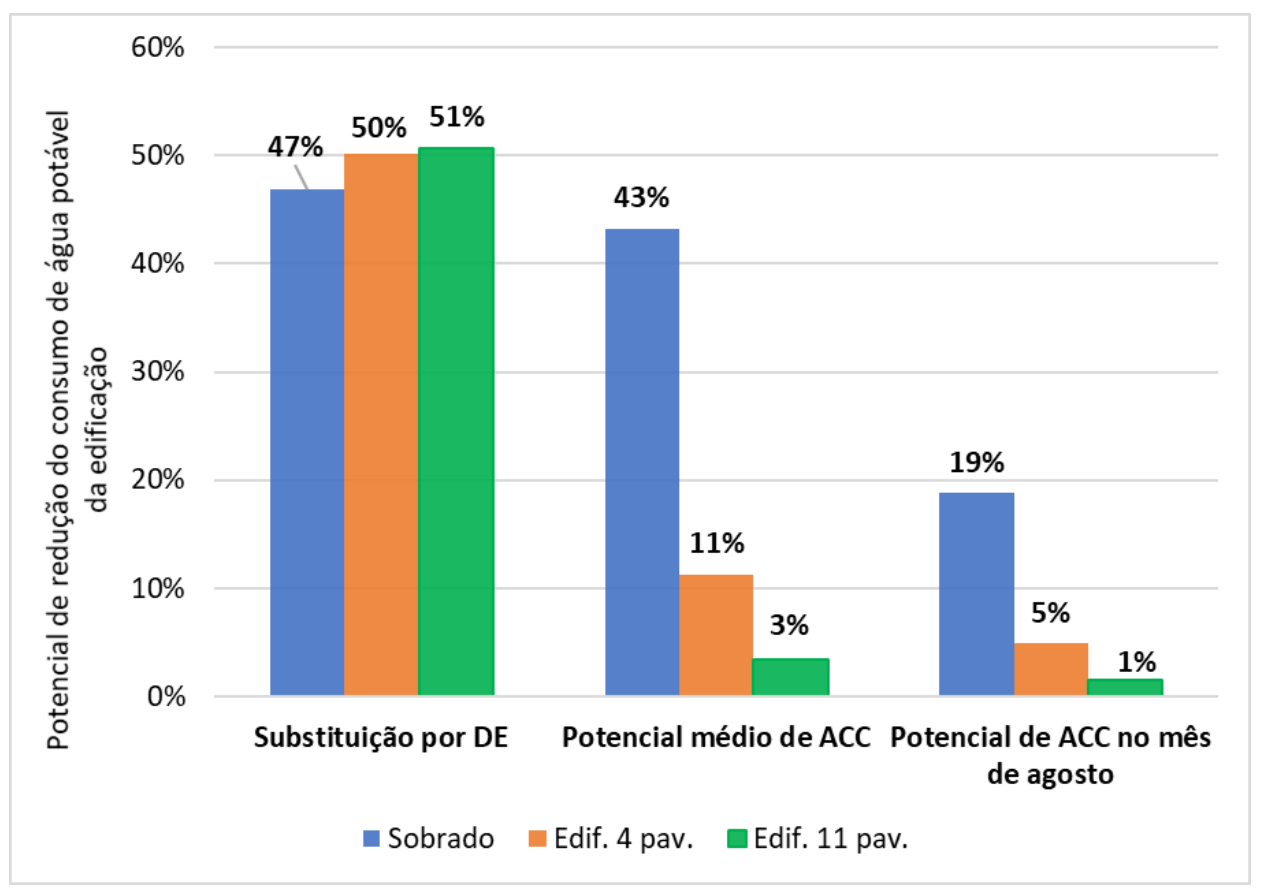

Fonte: Autoria própria (2021). 
Figura 3 - Potencial de redução do consumo de água potável por unidade residencial.

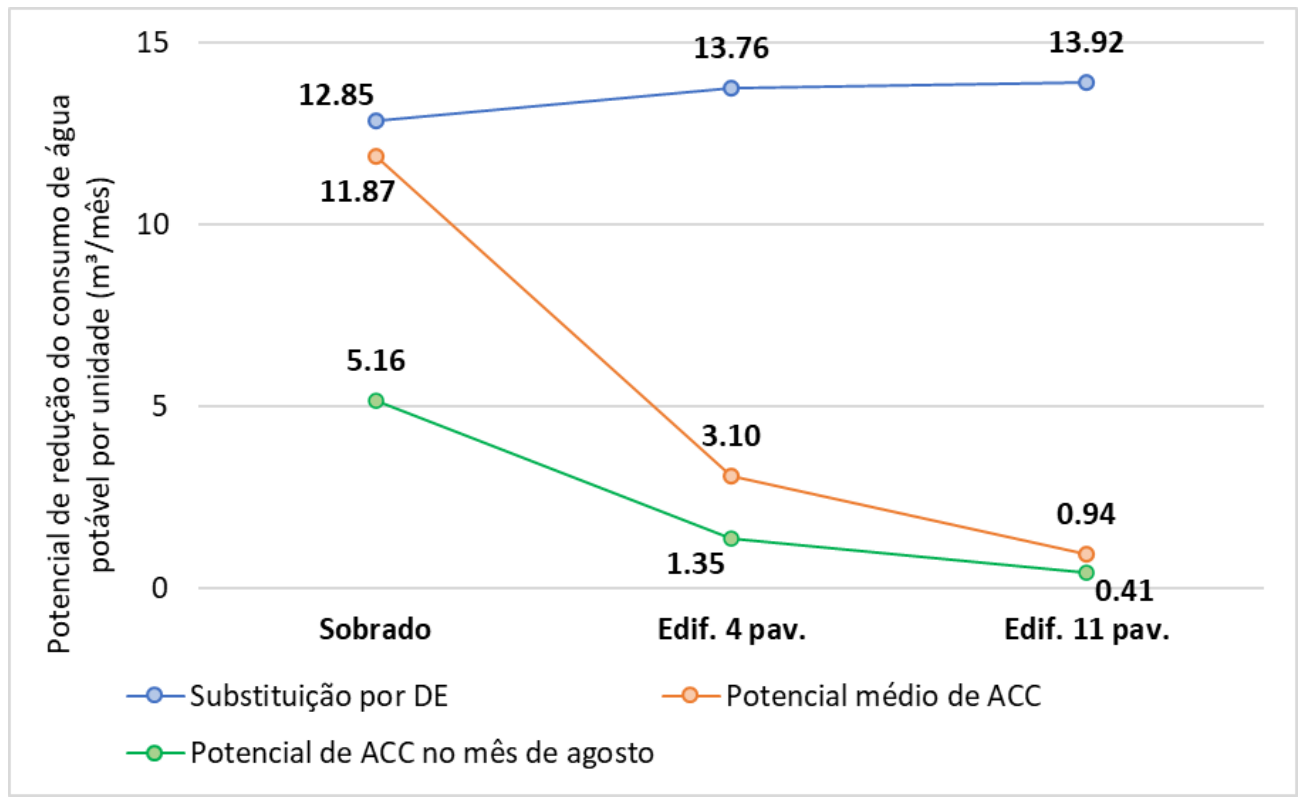

Fonte: Autoria própria (2021).

Esses resultados ressaltam a importância da gestão da demanda como o primeiro passo para a conservação da água na edificação. Apesar de ser uma ação bastante simples sob o ponto de vista construtivo, a substituição dos aparelhos tradicionais por DE por si só é capaz de reduzir o consumo de água de forma expressiva, refletindo esta economia na tarifa mensal. $A$ Tabela 2 apresenta o custo de compra e instalação dos DE e a economia anual decorrente da redução do consumo. Considerando as TAUR analisadas e os seus respectivos consumos, o tempo de retorno do investimento em DE é de, aproximadamente, 20 meses. Este tempo de retorno se iguala em todas as TAUR porque elas apresentam a mesma composição de áreas molhadas e, consequentemente, os mesmos DE por unidade residencial.

Tabela 2 - Investimento em DE, economia anual e tempo de retorno do investimento.

\begin{tabular}{l|c|c|c|c}
\hline \multicolumn{1}{c|}{ TAUR } & Economias & Instalação DE & Economia anual & Tempo de retorno (meses) \\
\hline Sobrado & 1 & $\mathrm{R} \$ 5.034,86$ & $\mathrm{R} \$ 3.046,67$ & 20 \\
\hline Edif. 4 pavtos. & 8 & $\mathrm{R} \$ 40.278,86$ & $\mathrm{R} \$ 24.373,40$ & 20 \\
\hline Edif. 11 pavtos. & 110 & $\mathrm{R} \$ 553.834,33$ & $\mathrm{R} \$ 335.134,21$ & 20 \\
\hline
\end{tabular}

Fonte: Autoria própria (2021).

Para edifícios novos, independentemente da TAUR, o uso de DE pode ser considerado já na fase de projeto e especificação dos componentes hidrossanitários, agregando características sustentáveis à edificação.

Como resultado complementar, a Figura 4 apresentada uma análise comparativa sobre o consumo de água em um edifício multifamiliar, considerando o uso de aparelhos tradicionais (AT) e o de DE. A partir da análise do gráfico, verifica-se que as distribuições de consumo dentro da unidade mudam e as referências antigas passam a não ser mais válidas. 
Figura 4 - Consumo residencial - comparativo entre AT x DE

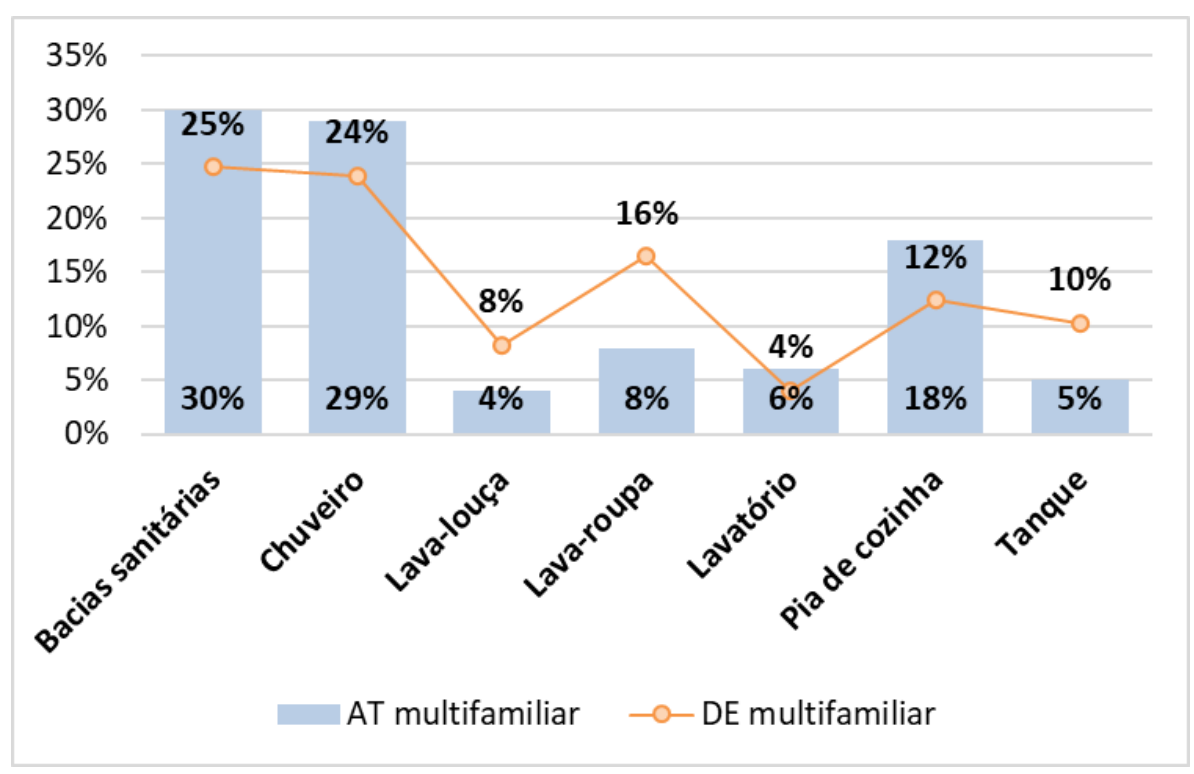

Fonte: Autoria própria (2021).

\section{CONCLUSÕES}

A troca dos aparelhos hidrossanitários pode ser uma solução sustentável generalizada na construção civil, tendo em vista que seus impactos positivos independem da TAUR. Por outro lado, o aproveitamento de ACC depende da área de captação e pode ser observado que o potencial de economia gerado por unidade domiciliar decresce com a verticalização da edificação.

Além disso, também foi possível observar que ao adotar os DE, as economias das TAUR avaliadas passam a se enquadrar na faixa mínima de consumo.

Por fim, o tempo de retorno de 20 meses, obtido para a substituição dos aparelhos tradicionais por DE, é aceitável para classe média brasileira, sendo necessário avaliar mecanismos de incentivo para classes menos favorecidas.

Como próxima etapa desta pesquisa, pretende-se fazer uma análise espacializada para toda a Bacia do Rio Trapicheiro. Nesta análise, cada tipologia arquitetônica aqui apresentada será readequada, se necessário, considerando a quantidade de banheiros informados no censo do IBGE. Além disso, as tipologias serão associadas à renda dos moradores e, assim, pretende-se estudar o impacto do investimento nos DE nas distintas classes sociais da bacia em questão.

\section{AGRADECIMENTOS}

Este trabalho foi desenvolvido no âmbito da Cátedra UNESCO “Drenagem Urbana em Regiões de Baixada Costeira", à qual os autores agradecem. 


\section{REFERÊNCIAS}

ABNT. NBR 15.527-Água da chuva: Aproveitamento de coberturas em áreas urbanas para fins não potáveis - Requisitos. Brasil: [s. n.], 2019a.

ABNT. NBR 16.782 - Conservação de água em edificações. Brasil: [s. n.], 2019b.

ABNT. NBR 16.783 - Uso de fontes não alterntivas em edificações. Brasil: [s. n.], 2019c.

ALERTA RIO. Dados pluviométricos. [s. d.]. Available at: http://www.sistema-alerta-rio.com.br/dadosmeteorologicos/download/dados-pluviometricos/. Acessado em: 3 ago. 2019.

BALOI, D. Sustainable construction: challenges and opportunities. 19th Annual ARCOM Conference, vol. 1, no. September, p. 289-297, 2003.

CEDAE. Companhia Estadual de Água e Esgoto: tarifas e consumo. [s. d.]. Disponível em: https://cedae.com.br/tarifas. Acessado em: 9 set. 2019.

CIB; UNEP-IETC. Agenda 21 for Sustainable Construction in Developing Countries. [S. I.: s. n.], 2002.

DA SILVA, A. O.; ARGÔLO, A. L.; DO VALE, M. B. R.; OKUMURA, C. K.; VERÓL, A. P. Proposta de Projeto de uma Edificação Sustentável Baseada no Conceito de Net Zero Water Buildings. Gestão e Gerenciamento, vol. 13, no. 13, p. 1-9, 2020. DOI 10.17648/nppg-gestaoegerenciamento-2447-1291v13-1. Disponível em: https://nppg.org.br/revistas/gestaoegerenciamento/article/view/498.

IBGE. Instituto Brasileiro de Geografia e Estatística: Censo 2010. [s. d.]. Disponível em: https://censo2010.ibge.gov.br/. Acessado em: 13 set. 2019.

PNCDA. DTA - Documento Técnico de Apoio n. ${ }^{\circ}$ B1 Elementos de análise econômica relativos ao consumo predial. Brasil: [s. n.], 1998.

QUEIROZ, D. M.; BLOOMFIELD, V. K.; REGO, A. Q.; FIGUEIREDO, N. O. Plano de integração áreas verdes: Tijuca e Vila Isabel. [S. I.: s. n.], 2008.

SALGADO, M. S. Projeto Integrado - Caminho para a produção de edificações sustentáveis: a questão dos Sistemas Prediais. 2008. XII Encontro Nacional de Tecnologia do Ambiente Construído [...]. [S. I.: s. n.], 2008. p. 11.

SNIS. Sistema Nacinal de Informações sobre Saneamento: Série histórica. [s. d.]. Disponível em: http://app4.mdr.gov.br/serieHistorica/. Acessado em: 23 abr. 2021. 